\title{
Dissipative dynamics in the massive boson limit of the sine-Gordon model
}

\author{
Ádám Bácsi, ${ }^{1,2,}$ * Cătălin Paşcu Moca, ${ }^{3,4}$ Gergely Zaránd, $, 3,5$ and Balázs Dóra ${ }^{1,6}$ \\ ${ }^{1}$ MTA-BME Lendület Topology and Correlation Research Group, \\ Budapest University of Technology and Economics, 1521 Budapest, Hungary \\ ${ }^{2}$ Department of Mathematics and Computational Sciences, \\ Széchenyi István University, 9026 Györ, Hungary \\ ${ }^{3}$ MTA-BME Quantum Dynamics and Correlations Research Group, \\ Budapest University of Technology and Economics, 1521, Budapest, Hungary \\ ${ }^{4}$ Department of Physics, University of Oradea, 410087, Oradea, Romania \\ ${ }^{5}$ BME-MTA Exotic Quantum Phases Research Group, Department of Theoretical Physics, \\ Budapest University of Technology and Economics, Budapest, Hungary \\ ${ }^{6}$ Department of Theoretical Physics, Budapest University of Technology and Economics, Budapest, Hungary
}

(Dated: August 13, 2021)

\begin{abstract}
We study the dissipative dynamics of one-dimensional fermions, described in terms of the sineGordon model in its massive boson or semi-classical limit, while keeping track of forward scattering processes. The system is prepared in the gapped ground state, and then coupled to environment through local currents within the Lindblad formalism. The heating dynamics of the system is followed using bosonization. The single particle density matrix exhibits correlations between the left and right moving particles. While the density matrix of right movers and left movers, is translationally invariant, the left-right sector is not, corresponding to a translational symmetry breaking charge density wave state. Asymptotically, the single particle density matrix decays exponentially with exponent proportional to $-\gamma t|x| \Delta^{2}$ where $\gamma$ and $\Delta$ are the dissipative coupling and the gap, respectively. The charge density wave order parameter decays exponentially in time with an interaction independent decay rate. The second Rényi entropy grows linearly with time and is essentially insensitive to the presence of the gap.
\end{abstract}

\section{INTRODUCTION}

Interacting quantum particles in one dimension typically either retain some low energy excitations and form a Luttinger liquid, or become gapped due to strong interactions and realize, e.g., an insulator. The latter phase is often described by the famous sine-Gordon model $[1,2]$ or by its close relative, the simpler massive boson model. The behavior of these systems is well documented in closed quantum systems [3, 4] in and out of equilibrium $[5,6]$, and faithfully realized in condensed matter, cold atom or other complex systems. In spite of its relevance, the response of these models to dissipative coupling to environment is much less studied and understood.

The interplay of dissipation and strong correlations, combined with reduced dimensionality [7-16], promises to provide a plethora of interesting phenomena [17-24]. This includes algebraic or exponential decay of correlation function in dissipative many-particle systems [8], universal features in the vaporization dynamics of Luttinger liquids[25] or black hole evaporation through Hawking radiation[26, 27]. In addition, propagators and correlation spreading also reveal unexpected features [12]. A recent experiment [28] on Josephson-coupled one dimensional bosons as well as related theoretical analysis[29] also hint to the importance of incorporating dissipation into sine-Gordon theory.

This motivates us to take a closer look at the dissipative dynamics of the sine-Gordon (sG) model. The sG model is Bethe ansatz solvable as a closed quantum system $[6,30]$, its solution simplifies, however, significantly at the so-called Luther-Emery line $[6,30]$ and also in the semi-classical [31] or massive boson limit, when solitons are neglected, and the interaction potential is expanded around one of its minima, reducing the model to the massive boson or Klein-Gordon model [31]. This 'massive boson' limit captures the basic correlations, and we focus on this limit to understand dissipative dynamics.

We consider spinless interacting fermions in the presence of a gap in their spectrum, arising due to backscattering or umklapp processes [6], or simply due to a staggered dimerizing deformation or potential of a one dimensional half-filled lattice. We assume a dissipation coupled to the current operator, and turn on dissipation at time $t=0$, as a certain kind of dissipative quantum quench. We use bosonization to investigate the spatial and temporal decay of the fermionic single particle density matrix

$$
G_{\alpha \beta}(x ; t) \equiv\left\langle\psi_{\alpha}^{+}(x) \psi_{\beta}(0)\right\rangle, \quad \alpha, \beta=L, R,
$$

with $\psi_{L}$ and $\psi_{R}$ referring to the left- and right moving fermionic fields (see Eq. (10)), respectively and the expectation value defined as $\langle\ldots\rangle=\operatorname{Tr}\{\rho(t) \ldots\}$, with $\rho(t)$ the time evolved density operator. We write the single particle density matrix as

$$
G_{\alpha \beta}(x ; t)=G_{\alpha \beta}(x ; 0) e^{F_{\alpha \beta}(x, t)},
$$

and determine the functions $F_{R R}(x, t)$ and $F_{L R}(x, t)$. In contrast to the dissipative Luttinger liquid case [25], the 
correlator between right and left moving fermions becomes finite due to the presence of the gap.

We find that in the long distance and long time limit, the single particle density matrices of both $G_{R R}$ and $G_{L R}$ decay exponentially with an exponent proportional to $-\gamma t|x| \Delta^{2}$ where $\gamma$ and $\Delta$ are the dissipative coupling and the gap, respectively. The von Neumann entropy of the system also grows similarly to the Luttinger liquid as $-t \ln (t)$, and is largely insensitive to the presence of the gap. These results are also relevant for dissipative interacting relativistic field theories, such as the massless Thirring model[1,2].

\section{MASSIVE BOSON LIMIT OF THE SINE-GORDON MODEL}

The one dimensional sine-Gordon (sG) model $[1,6,30$, 32] describes interacting fermions, bosons or spins [6]. The sG model is constructed in terms of the Bose field, $\phi(x)[6]$,

$$
\phi(x)=-i \sum_{q \neq 0} \sqrt{\frac{\pi|q|}{2 L}} \frac{1}{q} e^{-i q x}\left(b_{q}^{+}+b_{-q}\right)
$$

with $b_{q}$ denoting canonical bosonic operators of momentum $q$. It consists of the conventional quadratic Luttinger liquid Hamiltonian, $H_{L L}$, incorporating forward scattering processes, and an additional $-J \int d x \cos (2 \phi(x))$ perturbation. If the cosine term is relevant, it opens up a gap in the spectrum, and the field $\phi(x)$ gets locked into its minima.

This cosine term can arise due to strong umklapp scattering [6] or backscattering [2], or simply due to a staggered potential or dimerization [33], which can all open up a gap in the spectrum. For the sake of concreteness, we can consider a one dimensional tight binding, half filled lattice of spinless fermions, interacting via nearest neighbour interaction, in the presence of a staggered potential [6]. This maps onto the anisotropic XXZ Heisenberg chain in the presence of a staggered magnetic field in the $z$ direction [4]. Strong repulsive interaction without the staggered potential, or weak interactions in the presence of the staggered potential yield the sine-Gordon type low energy physics. Alternatively, one can consider a one dimensional p-wave superconductor in the presence of electron-electron interaction [34], though the cosine term would contain the dual field of $\phi(x)$.

Deep in the massive phase, one can approximate the cosine term as $\sim \int d x 2 J \phi^{2}(x)$ [31]. This represents the so-called semi-classical limit of the model, which is described by massive bosonic excitations [31]. In this limit, the Hamiltonian can be written in terms of the bosonic annihilation operators $b_{q}$ as

$H=\sum_{q>0}\left[\omega(q)\left(b_{q}^{+} b_{q}+b_{-q}^{+} b_{-q}\right)+g(q)\left(b_{q}^{+} b_{-q}^{+}+b_{q} b_{-q}\right)\right]$.

Here, $\omega(q)=\left(v_{0}+g_{4}\right) q+\Delta^{2} /\left(2 v_{0} q\right)$ and $g(q)=g_{2} q+$ $\Delta^{2} /\left(2 v_{0} q\right)$ with $v_{0}$ the bare sound velocity of the noninteracting and non-gapped system, $g_{2}$ and $g_{4}$ are the conventional forward scattering interactions $[6,35]$ and the energy scale $\Delta \sim 2 J$ is proportional to the gap or mass of the elementary excitations. Note that in the absence of $\Delta$, the model is identical to the interacting Luttinger model [25].

Since the Hamiltonian is quadratic in the bosonic operators, it can be diagonalized by Bogoliubov transformation leading to

$$
H=E_{G S}+\sum_{q>0} \tilde{\omega}(q)\left(B_{q}^{+} B_{q}+B_{-q}^{+} B_{-q}\right),
$$

where $\tilde{\omega}(q)=\sqrt{\omega^{2}(q)-g^{2}(q)}=\sqrt{(\tilde{v}|q|)^{2}+\tilde{\Delta}^{2}}$ is the gapped spectrum, and $E_{G S}=\sum_{q>0}(\tilde{\omega}(q)-\omega(q))$ is the ground state energy. Due to the interaction, the sound velocity and the gap are renormalized as $\tilde{v}=$ $\sqrt{\left(v_{0}+g_{4}\right)^{2}-g_{2}^{2}}$ and $\tilde{\Delta}=\Delta \sqrt{K \tilde{v} / v_{0}}$, where $K=$ $\sqrt{\left(v_{0}+g_{4}-g_{2}\right) /\left(v_{0}+g_{4}+g_{2}\right)}$ is the Luttinger parameter. For attractive or repulsive interactions, $K>1$ or $K<1$, respectively.

The gap $\tilde{\Delta}$ and the renormalized sound velocity, $\tilde{v}$, define a natural length scale, the coherence length,

$$
\tilde{\xi}=\tilde{v} / \tilde{\Delta} .
$$

As we shall see later, the coherence length $\tilde{\xi}$ represents a characteristic length scale that separates the short and the long distance regimes for the spatial dependence of the fermionic Green's functions $G(x ; t)$.

The goal of the present investigation is to study the non-equilibrium dynamics of the semi-classical or massive boson limit of the sine-Gordon model following a sudden quench at $t=0$ when the coupling to the environment is switched on. For the dynamics, the initial state is the ground state of Eq. (4), i.e., no bosonic excitations are present. After the quench, $t>0$, the time evolution of the system is governed by the quantum master equation of Lindblad type for the density operator

$$
\partial_{t} \rho=-i[H, \rho]+\gamma \int \mathrm{d} x([j(x), \rho j(x)]+h . c .)
$$

where $\rho$ is the density operator. In Eq. (6) $j(x)$ is the current operator, which is a natural choice of jump operator when the environment is a fluctuating vector potential or gauge field [10, 25, 36-39]. In the bosonization language, the current operator is expressed with the help 
of the bosonic annihilation operators [6] as

$$
j(x)=\sum_{q \neq 0} \sqrt{\frac{|q|}{2 \pi L}} \operatorname{sgn}(q) e^{-i q x}\left(b_{-q}-b_{q}^{+}\right),
$$

and the integral in Eq. (6) yields

$$
\partial_{t} \rho=-i[H, \rho]+\frac{\gamma}{2 \pi} \sum_{q \neq 0}\left(\left[L_{q}, \rho L_{q}^{+}\right]+\text {h.c. }\right)
$$

where $L_{q}=\sqrt{|q|}\left(b_{q}-b_{-q}^{+}\right)=\sqrt{|q|}\left(B_{q}-B_{-q}^{+}\right) / \sqrt{\mathcal{K}(q)}$ with $\mathcal{K}(q)=K \tilde{v}|q| / \tilde{\omega}(q)$.

The Lindblad equation allows us to calculate the expectation value of the occupation number of Bogoliubov bosons $B_{q}^{+} B_{q}$ and the anomalous operator, which are obtained analytically as

$$
\begin{gathered}
n_{q}^{B}(t)=\operatorname{Tr}\left[\rho(t) B_{q}^{+} B_{q}\right]=\frac{\gamma|q|}{\pi \mathcal{K}(q)} t \\
m_{q}^{B}(t)=\operatorname{Tr}\left[\rho(t) B_{q}^{+} B_{-q}^{+}\right]=\frac{\gamma}{2 i \pi \tilde{v} K}\left(e^{2 i \tilde{\omega}(q) t}-1\right) .
\end{gathered}
$$

The linear growth of the boson number implies that in the long time limit the system is heated up to infinite temperature. This feature is the same as found in the gapless Luttinger model [25, 40], and follows from the fact that the jump operator is hermitian.

Having discussed the properties of the bosonic operators, we return now to the original fermionic fields, which are probed experimentally. The physical fermions are decomposed into right and left going particles as

$$
\psi(x)=e^{i k_{F} x} \psi_{R}(x)+e^{-i k_{F} x} \psi_{L}(x),
$$

where $k_{F}$ is the Fermi wavenumber and $\psi_{R, L}(x)$ is the field operator for the right $(R)$ and left $(L)$ moving fermionic quasiparticles. These can be expressed in terms of the bosons as

$$
\psi_{R / L}=\frac{1}{\sqrt{2 \pi \alpha}} e^{i(\theta(x) \pm \phi(x))},
$$

with $\theta(x)$ the dual field $\phi(x)$ [6]. The relevance of the cosine term therefore implies the locking of the phase $\phi$, corresponding to a finite expectation value of $\psi_{L}^{+} \psi_{R}$, and a spatially modulated particle density.

The equal time single particle density matrix is defined as

$$
\begin{aligned}
& \mathcal{G}(x, y ; t)=\left\langle\psi^{+}(x) \psi(y)\right\rangle= \\
& =e^{i k_{F}(x+y)}\left\langle\psi_{L}^{+}(x) \psi_{R}(y)\right\rangle+\text { c.c. }+ \\
& \quad+e^{i k_{F}(y-x)}\left\langle\psi_{R}^{+}(x) \psi_{R}(y)\right\rangle+e^{i k_{F}(x-y)}\left\langle\psi_{L}^{+}(x) \psi_{L}(y)\right\rangle .
\end{aligned}
$$

Here, the expressions within the expectation values are translational invariant, i.e. depend only on $x-y$, while the exponential prefactors $\exp \left( \pm i k_{F}(x+y)\right)$ are not. In a Luttinger liquid without the sine-Gordon term, only the $R R$ and $L L$ correlators are finite, the $R L$ and $L R$ terms vanish identically. However, with the sine-Gordon term, these become also finite and need to be considered on equal footing.

The diagonal of the density matrix, $\mathcal{G}(x, x ; t)$, is just the particle density,. As stated earlier, for any finite $\left\langle\psi_{R}^{+}(x) \psi_{L}(x)\right\rangle \neq 0$ it contains spatially inhomogeneous part $\sim e^{i 2 k_{F} x}\left\langle\psi_{L}^{+}(x) \psi_{R}(x)\right\rangle+c . c$, and describes a charge density wave pattern.

\section{EQUAL-TIME SINGLE PARTICLE DENSITY MATRIX OF THE RIGHT MOVERS}

To have a deeper understanding about the heat up process of the fermions, it is worth studying the time evolution of the correlations. We investigate first the time dependence of the equal-time single particle density matrix of the right movers

$$
G_{R R}(x ; t)=\operatorname{Tr}\left\{\rho(t) \psi_{R}^{+}(x) \psi_{R}(0)\right\},
$$

with $\psi_{R}$ defined in Eq. (11). Later, we will also study the correlation between right- and left moving particles.

After a long algebraic derivation similar to the one in Ref. [41], the single particle density matrix is calculated as

$$
\ln \left(\frac{G_{R R}(x ; t)}{G_{0}(x)}\right)=-\sum_{q>0} \frac{8 \pi}{L q} n_{q}^{b}(t) \sin ^{2}\left(\frac{q x}{2}\right),
$$

where $G_{0}(x)=\frac{1}{2 \pi(\alpha-i x)}$ is the single-particle density matrix of the gapless, non-interacting system, and $n_{q}^{b}(t)=$ $\left\langle b_{q}^{+} b_{q}\right\rangle$ is the occupation number of the $b$ bosons. The short length scale $\alpha$, appearing in $G_{0}$ and (11), is introduced to regularize the momentum integral by $e^{-\alpha|q|}$. The asymptotic behavior of the non-interacting gapless correlation function obeys the well-known $\sim 1 / x$ powerlaw for $x \gg \alpha$. In our model, the single particle density matrix deviates from $G_{0}(x)$ due to the presence of both interaction and dissipation. The expectation value of the number of $b$-bosons is calculated as

$n_{q}^{b}(t)=\frac{\omega(q)}{\tilde{\omega}(q)} n_{q}^{B}(t)-\frac{g(q)}{\tilde{\omega}(q)} \operatorname{Re}\left(m_{q}^{B}(t)\right)+\frac{1}{2}\left(\frac{\omega(q)}{\tilde{\omega}(q)}-1\right)$,

in which the time dependence only occurs through the functions $n_{q}^{B}(t)$ and $m_{q}^{B}(t)$, defined in Eqs. (9). Here, the first two terms arise from dissipation, while the last one follows from quantum fluctuations due to interactions.

At $t=0$, i.e., when the system is in the vacuum of $B$-bosons, both $n_{q}^{B}$ and $m_{q}^{B}$ vanish, and the initial single particle density matrix is written as

$$
G_{R R}(x ; 0)=\frac{i}{2 \pi \alpha} \exp \left(-\sum_{q>0} \frac{4 \pi}{L|q|} \frac{\omega(q)}{\tilde{\omega}(q)} \sin ^{2}\left(\frac{q x}{2}\right)\right) .
$$


We use again the exponential cut-off with $\alpha$ to regularize the momentum integrals. For a small gap, $\tilde{\Delta} \ll \tilde{v} / \alpha$, i.e. $\alpha \ll \tilde{\xi}$, the $t=0$ single-particle density matrix can be calculated analytically as

$$
\begin{gathered}
G_{R R}(x ; 0)=\frac{i}{2 \pi \alpha} \exp \left(\frac{1}{2 K}\left(1+\frac{1}{2} G_{M}\left(\frac{x^{2}}{4 \tilde{\xi}^{2}}\right)\right)+\right. \\
\left.+\frac{K+K^{-1}}{2}\left(\ln \left(\frac{\alpha}{\tilde{\xi}}\right)-\frac{\pi}{2} \operatorname{Re} Y_{0}\left(\frac{i x}{\tilde{\xi}}\right)\right)\right)
\end{gathered}
$$

where $G_{M}(y)=G_{13}^{21}\left(y \mid \begin{array}{ccc}3 / 2 & \\ 0 & 1 & 1 / 2\end{array}\right)$ is a Meijer Gfunction[42] and $Y_{0}$ the Bessel function of the second kind. It is meaningful to extract the short and long distance behaviors, obtained as

$G_{R R}(x ; 0)=\frac{i}{2 \pi \alpha} \begin{cases}\left|\frac{\alpha}{x}\right|^{\left(K+K^{-1}\right) / 2}, & \alpha \ll x \ll \tilde{\xi}, \\ \left(\frac{\alpha}{\xi}\right)^{\left(K+K^{-1}\right) / 2} e^{-\frac{\pi}{4 K}\left|\frac{x}{\xi}\right|,} & \tilde{\xi} \ll x .\end{cases}$

For short distances, $x \ll \tilde{\xi}$, the gap has no effect on the dynamics and the power-law decay with the exponent $\left(K+K^{-1}\right) / 2$ is characteristic of the interacting, gapless Luttinger model. At long distances, $x \gg \tilde{\xi}$, however, the gap becomes relevant and the correlation function decays exponentially with $x$.

After switching on the coupling to the environment, the single particle density matrix is expected to decrease with time according to the intuition that the system is heated up to infinite temperature. The time-dependent part of the equal-time correlation function is obtained by substituting the time-dependent terms of Eqs. (14) into (13),

$$
\begin{aligned}
& F_{R R}(x, t) \equiv \ln \left(\frac{G_{R R}(x ; t)}{G_{R R}(x ; 0)}\right)= \\
& \quad=-\sum_{q>0} \frac{8 \pi}{L q}\left[\frac{\omega(q)}{\tilde{\omega}(q)} n_{q}^{B}(t)-\frac{g(q)}{\tilde{\omega}(q)} \operatorname{Re}\left(m_{q}^{B}(t)\right)\right] \sin ^{2}\left(\frac{q x}{2}\right) .
\end{aligned}
$$

The integral over $q$ can be calculated analytically only for the term containing $n_{q}^{B}(t)$. Using the exponential cut-off and taking the scaling limit, when $\alpha \ll\{x, \tilde{\xi}\}$, the integral yields the first term of Eq. (19), whose magnitude increases linearly with time.

The term containing $m_{q}^{B}(t)$ cannot be calculated analytically. Numerical investigation shows that this term features temporal oscillations for late times. These oscillations are captured analytically by approximating $\tilde{\omega}(q) \approx \tilde{\Delta}$. Together with the contribution of the $n_{q}^{B}(t)$ term, we get in the $\tilde{\Delta}^{-1} \ll t$ long time limit

$$
\begin{gathered}
F_{R R}(x, t) \approx-\frac{\gamma t}{\pi \alpha}\left(\frac{\pi \alpha|x|}{2 \tilde{\xi}^{2} K^{2}}+1+\frac{1}{K^{2}}\right)+ \\
+\frac{\gamma}{\tilde{\xi} K^{2}} \frac{\sin (2 \tilde{\Delta} t)}{2 \tilde{\Delta}}\left(\left|\frac{x}{2 \tilde{\xi}}\right|+\frac{\tilde{\xi}}{\pi \alpha}\left(1-K^{2}\right)\right) .
\end{gathered}
$$

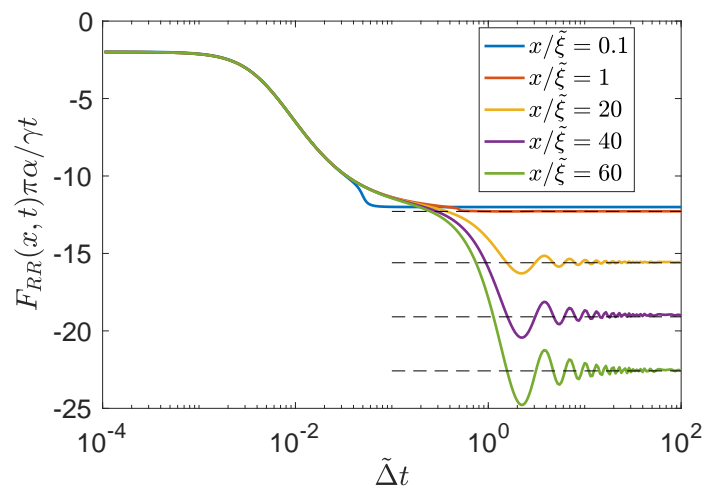

FIG. 1. Normalized effective decay rate of correlations in the $R R$ sector as a function of time at various distances for $K=0.3$ and $\alpha / \tilde{\xi}=0.01$. The curves are obtained by evaluating Eq. (18) numerically. They start from -2 in our dimensionless units for short times and reach the $x$ dependent asymptotic values (thin black dashed lines) from Eq. (20). In between, the crossover is described by Eq. (22).

For long times, the first term displaying the linear time dependence dominates in Eq.(19), and yields an exponential decay of the density matrix, the exponent of which decreases linearly with increasing separation $x$ (see Fig. 1). For times $\tilde{\Delta}^{-1} \lesssim t$ the second term yields oscillations of the exponent, but these oscillations fade away at longer times, and an exponential suppression with exponent

$$
F_{R R}(x, t) \approx-\frac{\gamma t}{\pi \alpha}\left(\frac{\pi \alpha|x|}{2 \tilde{\xi}^{2} K^{2}}+1+\frac{1}{K^{2}}\right)
$$

is found asymptotically for $t \gg \tilde{\Delta}^{-1}$.

In the opposite limit of extremely short times, $t \ll$ $\alpha / \tilde{v}$, i.e., for times shorter than the high energy time scale, however, we obtain an $x$ independent decay with an exponent

$$
F_{R R}(x, t) \approx-\frac{2 \gamma t}{\pi \alpha}, \quad t \ll \alpha / \tilde{v} .
$$

The richest behavior is found in the intermediate temporal region, $\alpha / \tilde{v} \ll t \ll 1 / \tilde{\Delta}$, where the first term in Eq. (18) gives results identical to those in Eq. (20). The second term in Eq. (18) with $m_{q}^{B}(t)$ gives, however, a correction for $2 \tilde{v} t \ll|x|$

$$
\begin{gathered}
\frac{G(x ; t)}{G(x ; 0)} \approx e^{-\frac{\gamma t}{2 K^{2} \xi}\left|\frac{x}{\xi}\right|} e^{-\frac{\gamma t\left(1+K^{2}\right)}{\pi \alpha K^{2}}} \times \\
\times \begin{cases}1, & 2 \tilde{v} t \gg|x|, \\
e^{-\frac{\gamma}{4 \tilde{v} K}\left(K-2 \frac{\tilde{v} t|x|}{K \xi^{2}}-\frac{1}{K}\right)}, & 2 \tilde{v} t \ll|x|,\end{cases}
\end{gathered}
$$

exhibiting very mild light cone behavior at $2 \tilde{v} t=x$, compared to the one found in closed quantum systems [31].

In the gapless case, $\Delta=0$, Eq. (22) renders the results obtained for dissipative Luttinger liquids [25]. Notice, 
however, that the intricate spatial and temporal behavior of Eq. (22) is valid only for $\alpha / \tilde{v} \ll t \ll 1 / \tilde{\Delta}$, and the late time, large distance behavior of the correlation function is dominated by the gap, giving rise to the unusual $\exp (-$ const $\cdot|x| t)$ decay of $G_{R R}(x ; t)$, one of the key findings of the present paper. This latter behavior starts to become dominant for $x \gtrsim \tilde{\xi}^{2} / \alpha$.

We compare the analytical estimates above with the numerical evaluation of Eq. (18) in Fig. 1, where we show the effective decay rate, $\sim \frac{1}{t} \ln \left[G_{R R}(x ; t) / G_{R R}(x ; t)\right]$ as a function of time. The spatial and temporal dependence of the single particle density matrix $G_{R R}(x ; t)$ agrees well with the analytical results, confirming the $\exp (-$ const $\cdot|x| t)$ asymptotics (horizontal dashed lines), while the early time decay rate remains largely insensitive to the spatial separation.

\section{CORRELATION BETWEEN LEFT- AND RIGHT MOVING PARTICLES}

While in the Luttinger liquid phase, left- and rightmovers are independent, they become correlated in the presence of a gap $[43,44]$, and the corresponding offdiagonal density operator

$$
G_{L R}(x ; t)=\operatorname{Tr}\left[\rho(t) \psi_{L}^{+}(x) \psi_{R}(0)\right]
$$

becomes finite. The trace is evaluated by using the bosonic representation, Eq. (11), similarly to the previous section. The decay factor of the single particle density matrix is obtained as

$$
\begin{aligned}
F_{L R}(x ; t) & \equiv \ln \left(\frac{G_{L R}(x ; t)}{G_{L R}(x ; 0)}\right)= \\
& =-\sum_{q>0} \frac{4 \pi}{L|q|}\left(n_{q}^{B}(t) \frac{\omega(q)-g(q) \cos (q x)}{\tilde{\omega}(q)}+\operatorname{Re}\left(m_{q}^{B}(t)\right) \frac{\omega(q) \cos (q x)-g(q)}{\tilde{\omega}(q)}-\operatorname{Im}\left(m_{q}^{B}(t)\right) \sin (q x)\right)
\end{aligned}
$$

which, interestingly, depends on time again only through the functions $n_{q}^{B}(t)$ and $m_{q}^{B}(t)$. The initial correlation function now reads

$$
G_{L R}(x ; 0)=\frac{1}{2 \pi \alpha} e^{-\sum_{q>0} \frac{2 \pi}{L|q|}\left[\mathcal{K}(q)+\frac{g(q)}{\tilde{\omega}(q)}(1-\cos (q x))\right]}
$$

and is evaluated as

$$
G_{L R}(x ; 0)=\frac{1}{2 \pi \alpha}\left(\frac{\alpha}{\tilde{\xi}}\right)^{\frac{1+K^{2}}{2 K}} \begin{cases}\left|\frac{\tilde{\xi}}{x}\right|^{\frac{1-K^{2}}{2 K}}, & \alpha \ll x \ll \tilde{\xi}, \\ e^{-\frac{\pi}{4 K}\left|\frac{x}{\tilde{\xi}}\right|}, & \alpha \ll \tilde{\xi} \ll x .\end{cases}
$$

Note that if the gap is zero, then $\tilde{\xi}$ is infinitely large, and the correlation function vanishes. Without forward scattering, $K=1, G_{L R}(x ; 0)$ stays constant within the coherence length, and vanishes exponentially outside it. The local density operator, $G_{L R}(0 ; t)$, characterizes the amplitude of the $2 k_{F}$ oscillating charge density profile. At the start of the dissipation quench and in the $\alpha \tilde{\Delta} \ll \tilde{v}$ limit we have

$$
G_{L R}(0 ; 0)=\frac{1}{2 \pi \alpha}\left(\frac{\alpha e^{\gamma_{E}} \tilde{\Delta}}{2 \tilde{v}}\right)^{K}
$$

with $\gamma_{E}=0.5772 \ldots$ denoting Euler's constant. In the absence of forward scattering interactions, $K=1$, the charge density wave amplitude $\left\langle\psi_{L}^{+}(x) \psi_{R}(0)\right\rangle$ is directly proportional to the gap, $G_{L R}(0 ; 0) \sim \Delta$, while this gets significantly renormalized due to interactions, $K \neq 1$, as
$\Delta \rightarrow \tilde{\Delta}^{K}$. The exponent $K$ is inherited from the scaling dimension of operator responsible for $2 k_{F}$ density fluctuations [6]. In particular, repulsive interactions with $K<1$ favor ordering by enhancing the value of the gap and also the amplitude of charge oscillations, while attractive interactions with $K>1$ reduce the gap, and suppress charge oscillations.

At long distances, the time-dependent part exhibits the same limiting behavior as the density matrix of the right movers,

$$
F_{L R}(x, t) \approx \begin{cases}-\frac{\gamma t}{\pi \alpha}\left(\frac{\pi \alpha|x|}{2 \tilde{\xi}^{2} K^{2}}+1+\frac{1}{K^{2}}\right), & t \gg 1 / \tilde{\Delta}, \\ -2 \frac{\gamma t}{\pi \alpha}, & t \ll \alpha / \tilde{v} .\end{cases}
$$

For long times and large distances, the gap thus dominates and leads to a $G_{L R} \sim e^{- \text {const. }|x| t}$ behavior, as also confirmed by the numerical evaluation of Eq. (24) (see Fig. 2).

At intermediate times, $\alpha / \tilde{v} \ll t \ll 1 / \tilde{\Delta}$, the decay is slightly different than the one obtained in the $R R$ and $L L$ sectors. We obtain, in particular

$$
\begin{aligned}
& \frac{G_{L R}(x ; t)}{G_{L R}(x ; 0)} \approx e^{-\frac{\gamma t}{2 K^{2} \tilde{\xi}}\left|\frac{x}{\tilde{\xi}}\right|} e^{-\frac{\gamma t\left(1+K^{2}\right)}{\pi \alpha K^{2}}} \times \\
& \times \begin{cases}e^{\frac{\gamma}{2 \tilde{v}}\left(\frac{\operatorname{sgn}(x)}{K}-1\right)}, & 2 \tilde{v} t \gg|x|, \\
e^{\frac{\gamma}{2 \tilde{v}}\left(\frac{1-K^{2}}{2 K^{2}}+\frac{\tilde{v} t x}{K^{2} \tilde{\xi}^{2}}\right)}, & 2 \tilde{v} t \ll|x|,\end{cases}
\end{aligned}
$$

which again features rather mild light cone effects. 


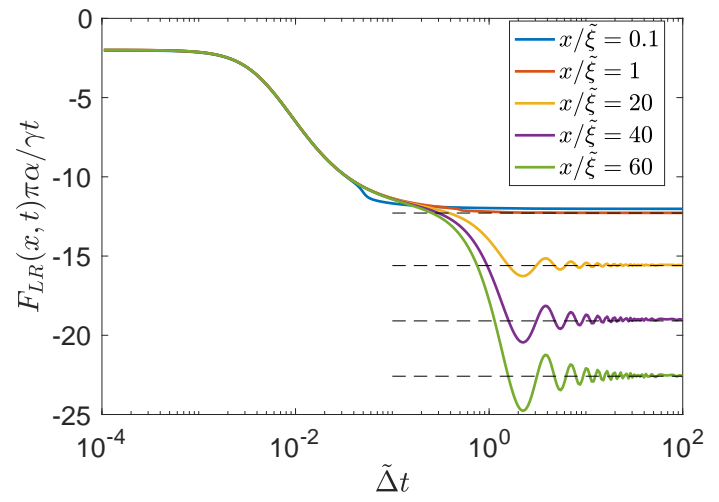

FIG. 2. Time-dependence of the decay rate of the anomalous $L R$ single particle density matrix, governing the amplitude of $2 k_{F}$ charge density wave correlations. The curves are obtained by evaluating Eq. (24) numerically with $K=0.3$ and $\alpha / \tilde{\xi}=$ 0.01 . Short and ling time asymptotics are very similar to those of the $R R$ sector (see in Fig. 1). The crossover is described by Eq. (29).

We can also follow the dissipation-induced temporal destruction of the $2 k_{F}$ charge density wave. The amplitude $\left\langle\psi_{L}^{+}(0) \psi_{R}(0)\right\rangle$ decays exponentially in time as

$$
\frac{G_{L R}(0 ; t)}{G_{L R}(0 ; 0)} \approx \begin{cases}e^{-\frac{4 \gamma t}{\pi \alpha}}, & t \ll \alpha / \tilde{v}, \\ e^{-\frac{2 \gamma t}{\pi \alpha}-\frac{\gamma}{2 v}}, & \alpha / \tilde{v} \ll t \ll 1 / \tilde{\Delta}, \\ e^{-\frac{2 \gamma t}{\pi \alpha}}, & 1 / \tilde{\Delta} \ll t\end{cases}
$$

with the decay rate reduced by a factor of 2 in course of the dissipative time evolution, as shown in Fig. 3.

\section{ENTANGLEMENT ENTROPY GENERATION}

The information loss in an open system is expressively demonstrated by the growth of the von Neumann entropy, defined as $S(t)=-\operatorname{Tr}[\rho(t) \ln \rho(t)]$. This latter can be regarded as the thermodynamical entropy, or as the measure of entanglement with the dissipative environment. Following the derivation of Ref. [25], the entropy

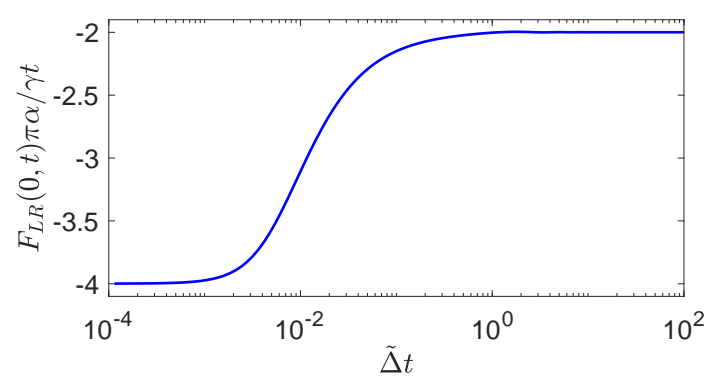

FIG. 3. Time-dependence of the decay rate of charge density wave order parameter for $\alpha \tilde{\Delta} / \tilde{v}=0.01$, (see Eq. (30)). Apart from the renormalization of its equilibrium value $\tilde{\Delta} \rightarrow \tilde{\Delta}^{K}$, the time dependence is completely independent of the forward scattering interaction. is obtained as

$$
S(t)=2 \sum_{q>0}\left[\left(N_{q}(t)+1\right) \ln \left(N_{q}(t)+1\right)-N_{q}(t) \ln N_{q}(t)\right]
$$

where $N_{q}(t)=\sqrt{\left(n_{q}^{B}(t)+\frac{1}{2}\right)^{2}-\left|m_{q}^{B}(t)\right|^{2}}-\frac{1}{2}$, with $n_{q}^{B}(t)$ and $m_{q}^{B}(t)$ the functions displayed in Eq. (9). For weak dissipation, i.e., when $\gamma \ll \tilde{v} K$, we get $N_{q}(t) \approx$ $\gamma \tilde{\omega}(q) t /(\tilde{v} K \pi)$ and the entropy is obtained as

$$
S(t) \approx \frac{L}{\pi \alpha}\left\{\begin{array}{cc}
-f(\alpha / \tilde{\xi}) \frac{\gamma t}{K \alpha \pi} \ln \frac{\gamma t}{K \alpha \pi}, & \gamma t \ll K \alpha \pi, \\
\ln \frac{\gamma t}{K \alpha \pi}, & \gamma t \gg K \alpha \pi
\end{array}\right.
$$

where $f(z)=\frac{\pi z}{2}\left(-Y_{1}(z)+\mathbf{H}_{1}(z)\right)$ with $Y_{1}$ the Bessel function of the second kind, and $\mathbf{H}_{1}$ is Struve function. In the scaling limit, we have $f(\alpha \ll \tilde{\xi})=1$, and the entropy exhibits the same time dependence as in the gapless case [25]. This behavior is explained by the fact that the entropy is mostly determined by high energy modes, and is not influenced by the presence or absence of the gap at low energies. We mention that for fermionic models with finite local Hilbert space, the entropy cannot be larger than $\sim L / \alpha$. Therefore, the second line in Eq. (32) could possibly show up in bosonic realization of the sineGordon models[5], where the local Hilbert space is much bigger.

Beside the von Neumann entropy, it is worth investigating the second Rényi entropy, which is more relevant experimentally [45-47]. The second Rényi entropy is defined as $S_{2}(t)=-\ln \operatorname{Tr}\left[\rho(t)^{2}\right]$, and is computed as

$$
S_{2}(t) \approx 2 \sum_{q>0} \ln \left(2 N_{q}(t)+1\right)
$$

In the weak dissipation limit, the early and long time dependence is calculated as

$$
S_{2}(t)=\frac{L}{\pi \alpha}\left\{\begin{array}{cc}
2 f\left(\frac{\alpha}{\xi}\right) \frac{\gamma t}{K \alpha \pi}, & \gamma t \ll K \alpha \pi, \\
\ln \frac{\gamma t}{K \alpha \pi}, & \gamma t \gg K \alpha \pi
\end{array}\right.
$$

which is again dominated by high energy modes and the gap does not affect the asymptotic temporal variations. Similar time dependencies are found in other systems as well[10].

\section{CONCLUSIONS}

We investigated the dissipative dynamics of the sineGordon model in its 'semi-classical' limit, where forward scattering interactions are still incorporated, but solitons are neglected, and the low energy Hamiltonian is described in terms of massive bosons. We considered the case of a current operator induced dissipation within the Lindblad formalism, where the dissipative dynamics 
remains still exactly solvable. We have focused, in particular, on the time evolution of the density operator of the underlying fermionic theory.

The interplay of forward scattering interaction, gap, and dissipation produces interesting features in the single particle density matrix. The density matrix of the right movers features Luttinger liquid type power law correlations within the coherence length, associated with the gap, $|x|<\tilde{\xi}$, but decays exponentially in space and time as $\sim \exp (-$ const $\cdot|x| t)$ in the long distance, late time limit.

More importantly, the anomalous density matrix between right and left moving fermions reveals a very similar spatio-temporal pattern. In the initial non-dissipative state, the bare gap $\Delta$ gets strongly renormalized by interactions, $\tilde{\Delta} \rightarrow \Delta^{K}$ with $K$ the Luttinger liquid parameter, and gets exponentially destroyed in time with a decay rate that gradually decreases by a factor of 2 .

While correlations and the single particle density matrix are very sensitive to the presence of the gap $\Delta$, the entropy is not. The von-Neumann and second Rényi entropies are extensive and grow initially as $-t \ln (t)$ and $t$, respectively due to the presence of interaction with the dissipative environment. This originates from the large number of high energy modes, which become populated fast due to heating from dissipation.

This research has been supported by the National Research, Development and Innovation Office - NKFIH within the Quantum Technology National Excellence Program (Project No. 2017-1.2.1-NKP-2017-00001), research projects K119442 and K134437, and within the Quantum Information National Laboratory of Hungary, and by the Romanian National Authority for Scientific Research and Innovation, UEFISCDI, under project no. PN-III-P4-ID-PCE-2020-0277.

* bacsi.adam@sze.hu

[1] S. Coleman, Quantum sine-gordon equation as the massive thirring model, Phys. Rev. D 11, 2088 (1975).

[2] W. E. Thirring, A soluble relativistic field theory, Ann. Phys. 3, 91 (1958).

[3] I. Kukuljan, S. Sotiriadis, and G. Takacs, Correlation functions of the quantum sine-gordon model in and out of equilibrium, Phys. Rev. Lett. 121, 110402 (2018).

[4] B. Bertini, D. Schuricht, and F. H. L. Essler, Quantum quench in the sine-gordon model, Journal of Statistical Mechanics: Theory and Experiment 2014(10), P10035 (2014).

[5] M. A. Cazalilla, R. Citro, T. Giamarchi, E. Orignac, and M. Rigol, One dimensional bosons: From condensed matter systems to ultracold gases, Rev. Mod. Phys. 83, 1405 (2011).

[6] T. Giamarchi, Quantum Physics in One Dimension (Oxford University Press, Oxford, 2004).

[7] J.-S. Bernier, R. Tan, C. Guo, C. Kollath, and D. Poletti,
Melting of the critical behavior of a tomonaga-luttinger liquid under dephasing, arXiv:2003.13809.

[8] Z. Cai and T. Barthel, Algebraic versus exponential decoherence in dissipative many-particle systems, Phys. Rev. Lett. 111, 150403 (2013).

[9] M. V. Medvedyeva, F. H. L. Essler, and T. Prosen, Exact bethe ansatz spectrum of a tight-binding chain with dephasing noise, Phys. Rev. Lett. 117, 137202 (2016).

[10] V. Alba and F. Carollo, Spreading of correlations in markovian open quantum systems, Phys. Rev. B 103, L020302 (2021).

[11] J.-S. Bernier, R. Tan, L. Bonnes, C. Guo, D. Poletti, and C. Kollath, Light-cone and diffusive propagation of correlations in a many-body dissipative system, Phys. Rev. Lett. 120, 020401 (2018).

[12] Y. Ashida and M. Ueda, Full-counting many-particle dynamics: Nonlocal and chiral propagation of correlations, Phys. Rev. Lett. 120, 185301 (2018).

[13] D. S. Kosov, T. Prosen, and B. Žunkovič, Lindblad master equation approach to superconductivity in open quantum systems, Journal of Physics A: Mathematical and Theoretical 44, 462001 (2011).

[14] M. H. Fischer, M. Maksymenko, and E. Altman, Dynamics of a many-body-localized system coupled to a bath, Phys. Rev. Lett. 116, 160401 (2016).

[15] A. J. Daley, Quantum trajectories and open many-body quantum systems, Advances in Physics 63, 77 (2014).

[16] Y. Ashida, Z. Gong, and M. Ueda, Non-hermitian physics, Adv. Phys. 69(3), 249 (2020).

[17] S. Diehl, A. Micheli, A. Kantian, B. Kraus, H. P. Büchler, and P. Zoller, Quantum states and phases in driven open quantum systems with cold atoms, Nat. Phys. 4, 878 (2008).

[18] H. Pichler, A. J. Daley, and P. Zoller, Nonequilibrium dynamics of bosonic atoms in optical lattices: Decoherence of many-body states due to spontaneous emission, Phys. Rev. A 82, 063605 (2010).

[19] J. T. Barreiro, M. Müller, P. Schindler, D. Nigg, T. Monz, M. Chwalla, M. Hennrich, C. F. Roos, P. Zoller, and R. Blatt, An open-system quantum simulator with trapped ions, Nature 470, 486 (2011).

[20] B. Buca, J. Tindall, and D. Jaksch, Non-stationary coherent quantum many-body dynamics through dissipation, Nat. Commun. 10, 1730 (2019).

[21] M. Naghiloo, M. Abbasi, Y. N. Joglekar, and K. W. Murch, Quantum state tomography across the exceptional point in a single dissipative qubit, Nat. Phys. 15, 1232 (2019).

[22] N. Shibata and H. Katsura, Dissipative spin chain as a non-hermitian kitaev ladder, Phys. Rev. B 99, 174303 (2019).

[23] Y. Ashida, K. Saito, and M. Ueda, Thermalization and heating dynamics in open generic many-body systems, Phys. Rev. Lett. 121, 170402 (2018).

[24] C.-E. Bardyn, M. A. Baranov, C. V. Kraus, E. Rico, A. İmamoğlu, P. Zoller, and S. Diehl, Topology by dissipation, New Journal of Physics 15, 085001 (2013).

[25] A. Bácsi, C. P. Moca, G. Zaránd, and B. Dóra, Vaporization dynamics of a dissipative quantum liquid, Phys. Rev. Lett. 125, 266803 (2020).

[26] S. W. Hawking, Black hole explosions?, Nature 248, 30 (1974).

[27] R. Parentani and P. Spindel, Hawking radiation, Schol- 
arpedia 6, 6958 (2011).

[28] M. Pigneur, T. Berrada, M. Bonneau, T. Schumm, E. Demler, and J. Schmiedmayer, Relaxation to a phaselocked equilibrium state in a one-dimensional bosonic josephson junction, Phys. Rev. Lett. 120, 173601 (2018).

[29] D. X. Horváth, I. Lovas, M. Kormos, G. Takács, and G. Zaránd, Nonequilibrium time evolution and rephasing in the quantum sine-gordon model, Phys. Rev. A 100, 013613 (2019).

[30] A. O. Gogolin, A. A. Nersesyan, and A. M. Tsvelik, Bosonization and Strongly Correlated Systems (Cambridge University Press, Cambridge, 1998).

[31] A. Iucci and M. A. Cazalilla, Quantum quench dynamics of the sine-gordon model in some solvable limits, New J. Phys. 12, 055019 (2010).

[32] F. H. L. Essler and R. M. Konik, Application of massive integrable quantum field theories to problems in condensed matter physics (World Scientific, 2005), p. 684.

[33] In this case, the factor of 2 would be absent from the cosine term as $\cos (\phi(x))$.

[34] S. Gangadharaiah, B. Braunecker, P. Simon, and D. Loss, Majorana edge states in interacting one-dimensional systems, Phys. Rev. Lett. 107, 036801 (2011).

[35] J. Sólyom, The fermi gas model of one-dimensional conductors, Adv. Phys. 28, 201 (1979).

[36] V. Eisler, Crossover between ballistic and diffusive transport: the quantum exclusion process, Journal of Statistical Mechanics: Theory and Experiment 2011, P06007 (2011).

[37] K. Temme, M. M. Wolf, and F. Verstraete, Stochastic exclusion processes versus coherent transport, New Journal of Physics 14, 075004 (2012).
[38] A. Pereverzev and E. R. Bittnera, Quantum transport in chains with noisy off-diagonal couplings, J. Chem. Phys. 123, 244903 (2005).

[39] B. Horstmann, J. I. Cirac, and G. Giedke, Noise-driven dynamics and phase transitions in fermionic systems, Phys. Rev. A 87, 012108 (2013).

[40] M. Buchhold and S. Diehl, Nonequilibrium universality in the heating dynamics of interacting luttinger liquids, Phys. Rev. A 92, 013603 (2015).

[41] A. Bácsi, C. P. Moca, and B. Dóra, Dissipation-induced luttinger liquid correlations in a one-dimensional fermi gas, Phys. Rev. Lett. 124, 136401 (2020).

[42] I. S. Gradshteyn and I. M. Ryzhik, Table of Integrals, Series, and Products (Academic Press, New York, 2007).

[43] G. Grüner, Density waves in solids (Addison-Wesley, Reading, 1994).

[44] D. Orgad, Spectral functions for the tomonaga-luttinger and luther-emery liquids, Phil. Mag. B 81, 377 (2001).

[45] R. Islam, R. Ma, P. M. Preiss, M. E. Tai, A. Lukin, M. Rispoli, and M. Greiner, Measuring entanglement entropy in a quantum many-body system, Nature 528, 77 (2015).

[46] J. Li, R. Fan, H. Wang, B. Ye, B. Zeng, H. Zhai, X. Peng, and J. Du, Measuring out-of-time-order correlators on a nuclear magnetic resonance quantum simulator, Phys. Rev. X 7, 031011 (2017).

[47] T. Brydges, A. Elben, P. Jurcevic, B. Vermersch, C. Maier, B. P. Lanyon, P. Zoller, R. Blatt, and C. F. Roos, Probing rényi entanglement entropy via randomized measurements, Science 364(6437), 260 (2019). 